\title{
Crystal structure data
}

Table S1. Crystal data for (-) - mSNA-2

$\mathrm{C}_{12} \mathrm{H}_{19} \mathrm{NOS}$

$M_{r}=225.35$

Orthorhombic, $P 2_{1} 2_{1} 2_{1}$

$a=5.7913$ (19) ^

$b=12.976(3) \AA$

$c=17.478(4) \AA$

$V=1313.4(6) \AA^{3}$

$Z=4$
$D_{x}=1.140 \mathrm{~g} \mathrm{~cm}^{-3}$

$\mathrm{Cu} K \alpha$ radiation

Cell parameters from 25 reflections

$\theta=15.9-29.4^{\circ}$

$\mu=1.99 \mathrm{~mm}^{-1}$

$T=296$ (2) K

Plate, colourless

$0.22 \times 0.15 \times 0.07 \mathrm{~mm}$

Table S2. Data collection

Enraf-Nonius CAD-4 diffractomer

$$
\begin{aligned}
& R_{\text {int }}=0.074 \\
& \theta_{\max }=75.0^{\circ} \\
& h=0 \rightarrow 7 \\
& k=-16 \rightarrow 16 \\
& l=0 \rightarrow 21
\end{aligned}
$$

$\omega / 2 \theta$ scan

$T_{\min }=0.668, T_{\max }=0.873$

3051 measured reflections

2699 independent reflections

2654 reflections with $I>2 \sigma(I)$

every $60 \mathrm{~min}$

Table S3. Refinement

Refinement on $F^{2}$

$R\left[F^{2}>2 \sigma\left(F^{2}\right)\right]=0.048$

$w R\left(F^{2}\right)=0.131$

$S=1.08$

2699 reflections

145 parameters

Mixture of independent and constrained $\mathrm{H}$-atom refinement
Calculated weights $\quad w=1 /\left[\sigma^{2}\left(F_{o}^{2}\right)+(0.1015 P)^{2}+\right.$ $0.0902 P]$ where $P=\left(F_{o}^{2}+2 F_{c}^{2}\right) / 3$

$(\Delta / \sigma)_{\max }=0.001$

$\Delta \rho_{\max }=0.56 \mathrm{e}^{-3}$

$\Delta \rho_{\min }=-0.35$ e $\AA^{-3}$

Extinction correction: SHELXL

Extinction coefficient: 0.025 (2)

Absolute structure: Flack H D (1983), Acta Cryst. A39, 876-881

Flack parameter: $0.00(2)$ 
Table S4. Geometric parameters $\left(\AA ̊{ }^{\circ}\right)$

$\begin{array}{llllllll}\mathrm{S} 1 & - & \mathrm{O} 1 & 1.486(2) & \mathrm{C} 5 & - & \mathrm{C} 7 & 1.524(3) \\ \mathrm{S} 1 & - & \mathrm{N} 1 & 1.661(2) & \mathrm{C} 7 & - & \mathrm{C} 8 & 1.387(3) \\ \mathrm{S} 1 & - & \mathrm{C} 1 & 1.842(2) & \mathrm{C} 7 & - & \mathrm{C} 12 & 1.375(3) \\ \mathrm{N} 1 & - & \mathrm{C} 5 & 1.480(2) & \mathrm{C} 8 & - & \mathrm{C} 9 & 1.389(3) \\ \mathrm{C} 1 & - & \mathrm{C} 2 & 1.529(3) & \mathrm{C} 9 & - & \mathrm{C} 10 & 1.378(5) \\ \mathrm{C} 1 & - & \mathrm{C} 3 & 1.518(3) & \mathrm{C} 10 & - & \mathrm{C} 11 & 1.372(5) \\ \mathrm{C} 1 & - & \mathrm{C} 4 & 1.519(3) & \mathrm{C} 11 & - & \mathrm{C} 12 & 1.393(3) \\ \mathrm{C} 5 & - & \mathrm{C} 6 & 1.516(3) & & & & \end{array}$

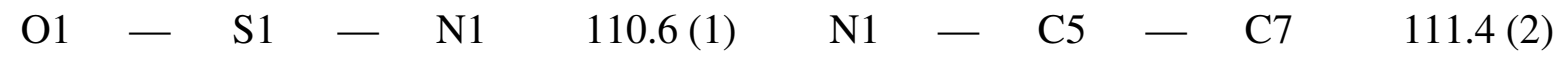

$$
\begin{aligned}
& \mathrm{O} 1-\mathrm{S} 1-\mathrm{C} 1 \quad 105.8(1) \quad \mathrm{C} 6-\mathrm{C}_{1}-\mathrm{C} 7 \quad 110.4(2)
\end{aligned}
$$

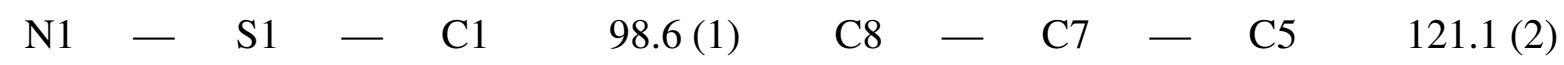

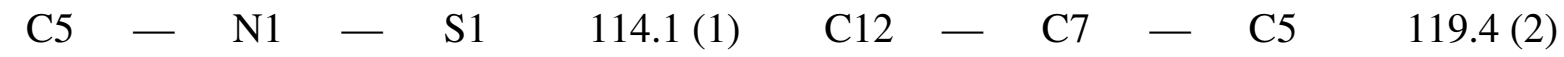

$$
\begin{aligned}
& \mathrm{C} 2-\mathrm{C} 1-\mathrm{S} 1 \quad \begin{array}{lllllll}
104.2(2) & \mathrm{C} 12 & - & \mathrm{C} 7 & - & \mathrm{C} 8 & 119.3(2)
\end{array}
\end{aligned}
$$

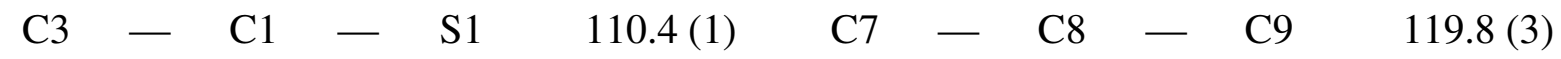

$$
\begin{aligned}
& \mathrm{C} 4-\mathrm{C} 1-\mathrm{S} 1 \quad 107.3(2) \quad \mathrm{C} 10-\mathrm{C} 9-\mathrm{C} 8 \quad 120.5(3) \\
& \mathrm{C} 3-\mathrm{C} 1-\mathrm{C} 2 \quad 111.4(2) \quad \mathrm{C} 11-\mathrm{C} 10-\mathrm{C} 9 \quad 119.9(2) \\
& \mathrm{C} 3-\mathrm{C} 1-\mathrm{C} 4 \quad 112.4(2) \quad \mathrm{C} 10-\mathrm{C} 11-\mathrm{C} 12 \quad 119.7(3) \\
& \mathrm{C} 4-\mathrm{C} 1-\mathrm{C} 2 \quad 110.8(2) \quad \mathrm{C} 7-\mathrm{C} 12-\mathrm{C} 11-120.8(3) \\
& \mathrm{N} 1-\mathrm{C} 5-\mathrm{C} 6 \quad 107.9(2) \\
& \mathrm{O} 1-\mathrm{S} 1-\mathrm{N} 1-\mathrm{C} 5-87.7(2) \quad \mathrm{C} 6-\mathrm{C} 5-\mathrm{C} 7-\mathrm{C} 12-99.9(3) \\
& \mathrm{C} 1-\mathrm{S} 1-\mathrm{N} 1-\mathrm{C} 5 \quad 161.7(2) \quad \mathrm{N} 1-\mathrm{C} 5-\mathrm{C} 7-\mathrm{C} 8-44.6(3) \\
& \mathrm{O} 1-\mathrm{S} 1-\mathrm{C} 1-\mathrm{C} 3-56.5(2) \quad \mathrm{C} 6-\mathrm{C} 5-\mathrm{C} 7-\mathrm{C} 8 \quad 75.2(3) \\
& \mathrm{N} 1-\mathrm{S} 1-\mathrm{C} 1-\mathrm{C} 3-57.8(2) \quad \mathrm{C} 12-\mathrm{C} 7-\mathrm{C} 8-\mathrm{C} 9-0.9(3) \\
& \mathrm{O} 1-\mathrm{S} 1-\mathrm{C} 1-\mathrm{C} 4-179.3(2) \quad \mathrm{C} 5-\mathrm{C} 7-\mathrm{C} 8-\mathrm{C} 9-176.0(2) \\
& \mathrm{N} 1-\mathrm{S} 1-\mathrm{C} 1-\mathrm{C} 4-64.9(2) \quad \mathrm{C} 7-\mathrm{C} 8-\mathrm{C} 9-\mathrm{C} 10 \quad 1.7(4) \\
& \mathrm{O} 1-\mathrm{S} 1-\mathrm{C} 1-\mathrm{C} 2-63.2(2) \quad \mathrm{C} 8-\mathrm{C} 9-\mathrm{C} 10-\mathrm{C} 11-1.3(5) \\
& \mathrm{N} 1-\mathrm{S} 1-\mathrm{C} 1-\mathrm{C} 2 \quad 177.5(1) \quad \mathrm{C} 9-\mathrm{C} 10-\mathrm{C} 11-\mathrm{C} 12-0.2(5) \\
& \mathrm{S} 1-\mathrm{N} 1-\mathrm{C} 5-\mathrm{C} 6 \quad 160.3(2) \quad \mathrm{C} 8-\mathrm{C} 7-\mathrm{C} 12-\mathrm{C} 11-0.2(4) \\
& \mathrm{S} 1-\mathrm{N} 1-\mathrm{C} 5-\mathrm{C} 7-78.4(2) \quad \mathrm{C} 5-\mathrm{C} 7-\mathrm{C} 12-\mathrm{C} 11 \quad 175.0(2) \\
& \mathrm{N} 1-\mathrm{C} 5-\mathrm{C} 7-\mathrm{C} 12 \quad 140.3(2) \quad \mathrm{C} 10-\mathrm{C} 11-\mathrm{C} 12-\mathrm{C} 7 \quad 0.5(4)
\end{aligned}
$$


Table S5. Hydrogen-bond parameters $\left(\AA{ }^{\circ}{ }^{\circ}\right)$

$\begin{array}{lcccc} & D-\mathrm{H} & \mathrm{H} . . . A & \text { D...A } & D-\mathrm{H} \ldots A \\ \mathrm{C} 3-\mathrm{H} 3 \mathrm{C} \ldots \mathrm{O} 1^{\mathrm{i}} & 0.96 & 2.59 & 3.474(3) & 153.8 \\ \mathrm{~N} 1-\mathrm{H} 1 \ldots \mathrm{O} 1^{\mathrm{ii}} & 0.83(3) & 2.32(3) & 3.132(2) & 166(3)\end{array}$

Symmetry codes: (i) $-1+x, y, z$; (ii) -1/2+x, 3/2-y, 2-z. 